\title{
A Novel Algorithm for Important Email Network Nodes Mining
}

\author{
Junkai $\mathrm{Yi}^{1}$, Zhirong $\mathrm{Li}^{1}$ and Xianghui Zhao ${ }^{2, *}$ \\ ${ }^{1}$ College of Information Science and Technology, Beijing University of Chemical Technology, Beijing 100029, P.R. of China \\ ${ }^{2}$ China information technology security evaluation center, Beijing 100085, P.R. of China \\ *Corresponding author
}

\begin{abstract}
Mining important persons is significant to network security, especially researches on email network centralization nowadays. To solve the problem that traditional PageRank algorithm is sensitive to the network disturbance, this paper proposes a method which decomposes email network into different layers based on the core number and eliminates the interferential nodes from outer layers. Besides, it improves PageRank algorithm which ranks the nodes quantitatively to find the important nodes. The experiments indicate that it improves the accuracy and reduces the computational complexity in mining important nodes from email network.
\end{abstract}

Keywords-nodes mining; core number; PAGERANK

\section{INTRODUCTION}

Mining important nodes is valuable. Mining essential persons in criminal network is also significant [1].

Email network centralization index includes degree centrality, betweenness centrality, closeness centrality, k-shell, etc[2].People propose some link mining algorithms based on centralization index, among which HITS and PageRank are popular. HITS is easy to be affected by irrelevant links. PageRank sorts webs and is more accurate. Li et al. propose a cost sensitive decision tree algorithm in data mining [3]. S.Huang et al. use Shannon-Parry Measure to evaluate nodes quantitatively [4]. Lu Zhong et al. propose Multiple Attribute Fusion to identify influential nodes [5]. Teng Wang et al. provide a ranking algorithm based on topological structure [6]. Liang Sun et al. propose comprehensive measure model (CMM) [7]. Kazumi Saito et al. propose super-mediators to mine influential nodes [8].

There are still problems in mining important nodes due to diverse index. This paper combines core number and degree centrality and improves PageRank algorithm.

\section{RELATED WORKS}

People propose many methods of mining important nodes. Wu et al. define function to mine important communities [9]. Jitesh Shetty et al. present an information theoretic model tested successfully on Enron email dataset [10]. Peter Lofgren and Ashish Goel improve PageRank algorithm which is more effective than the former [11]. Jessica Liebig and Asha Rao define a clustering coefficient [12].

\section{Evaluating Model of EMAil NetWork CENTRALIZATION}

\section{A. Adjacency Matrix}

Email network analyzed here is weighted directed network and can be expressed as adjacency matrix $A=\left(a_{i j}\right)_{N \times N}$. The element $a_{i j}$ in i-th row and j-th column is defined as:

$$
a_{i j}=\left\{\begin{array}{c}
w_{i j}, \text { If there is edge weighted } w_{i j} \text { drew from node } i \text { to node } j \\
0, \text { If there is no edge drew from node } i \text { to node } j
\end{array}\right.
$$

\section{B. Degree}

Degree of a node includes out-degree and in-degree. $k_{i}^{\text {out }}$ is out-degree and represents number of edges drew from node $i$ to other nodes. $k_{i}^{i n}$ is in-degree and means number of edges drew from other nodes to node $i$.

$$
k_{i}^{\text {out }}=\sum_{j=1}^{N} a_{i j}, \quad k_{i}^{i n}=\sum_{j=1}^{N} a_{j i} .
$$

\section{Centralization Index}

This paper describes network and its attributes using core number and degree centrality respectively.

1) Definition 1 ( $k$-core): K-core of a graph refers to subgraph after deleting nodes whose degrees are less than or equal to $\mathrm{k}$ and edges linked to them. The weighted directed graph is regarded as undirected graph in k-core decomposition here.

2) Definition 2 (Core Number): Core number of a node is the deepest core which contains it. If the core number of a node is $\mathrm{k}$, it belongs to $\mathrm{k}$-core rather than $(\mathrm{k}+1)$-core.

3) Definition 3 (Degree Centrality): A node with large degree is possibly important. The degree of a node in a network containing $\mathrm{N}$ nodes is less than $\mathrm{N}-1$.To normalize centralization index, degree centrality of a node whose degree is $\mathrm{ki}$ is defined as: 


$$
C_{k}=\frac{k_{i}}{N-1}
$$

\section{Algorithm OF Mining IMPORTANT NODES In EMAIL NETWORK}

\section{A. Optimize Email Network Using Core Number of Nodes}

A node of a network with large degree may have big core number $k_{i}$ and be in inner core. Or it may have small core number $k_{i}$ and be in outer core. In email network, a node with large degree is probably not an important node. Enlarging threshold of core number gradually, deleting interferential nodes whose degrees are large from outer network and optimizing the structure of network can reduce computational complexity of PageRank algorithm.

\section{Algorithm 1 core number compute algorithm}

Input: email network matrix $A$

Output: core number of every node core[i]

1) for (every node $i$ in $A$ ) do

2) if degrees[i] $<=$ core then //judge whether node's degree is less than or equal to core or not

3) degrees[i]=-1;//if it is, its core number is core and delete it

4) nbr=nt.n_neighbors(i);//find neighboring nodes of node $i$

5) degrees[nbr[j]]--;//subtract one from degree of all the neighboring nodes

6) end if

7) core++;

8)end for

9)for (every node $i$ in $A$ ) do

10) return core[i];

11)end for

B. Compute PR Values of Nodes Using PageRank Algorithm

Importance of a node on the network depends on quantity and quality of other nodes which point to it. In general directed network, basic PageRank algorithm can be described as following:

(1) Initial step: preset initial PageRank values of all nodes as $P R_{i}(0), i=1,2, \ldots, N$. Which satisfy the condition: $\sum_{i=1}^{N} P R_{i}(0)=1$.

(2) Compute $P R$ values:

$$
P R_{i}(k)=\sum_{j=1}^{N} a_{j i} \frac{P R_{j}(k-1)}{k_{j}^{\text {out }}}, i=1,2, \ldots, N
$$

In basic PageRank algorithm, if the information flow reaches a node whose out-degree is 0 , it won't go to other nodes. So scale constant $\mathrm{s} \in(0,1)$ is introduced to adjust $P R$ values. Initial step stays the same and the equation of computing $P R$ values changes into:

$$
P R_{i}(k)=s \sum_{j=1}^{N} a_{j i} \frac{P R_{j}(k-1)}{k_{j}^{\text {out }}}+\frac{1-s}{N}, i=1,2, \ldots, N
$$

Traditional PageRank algorithm is sensitive to interference of the network:

(1) Nodes with large $P R$ values are more likely to cite other nodes rather than being cited.

(2) Nodes with large $P R$ values often cite important nodes. On the contrary, nodes with small values cite general nodes more often.

$a_{v}$ is introduced as adjusted variable. The computing steps are shown as following:

Step 1 Divide the number of reverse links of node $j$ by forward links, that is $I O_{j}=\frac{I N_{j}}{O U T_{j}} \cdot I N_{j}$ and $O U T_{j}$ represent respectively $P R$ values passed from other nodes to node $j$ and from node $j$ to other nodes. The $I O$ values decide the possibility of getting $P R$ values from network.

Step 2 Assume nodes which point to node $j$ are $i_{1}, i_{2}, \ldots i_{n}$. The numbers of reverse links of nodes pointed from node $i$ are respectively $I N_{1}, I N_{2}, \ldots I N_{n}$. The numbers of forward links are $O U T_{1}, O U T_{2}, \ldots O U T_{n}$. And the ratios are respectively $I O_{1}, I O_{2}, \ldots I O_{n}$. Distribute $P R$ values as:

$$
a_{v}=a_{j}=\frac{I O_{j}}{\sum_{i=1}^{n} I O_{i}}
$$

This paper redefines important members. The important node must suffice three conditions simultaneously: (1)it's cited by important nodes. (2)it's cited by many nodes. (3)it cites other nodes much less than being cited. Improved PageRank algorithm remains the initial step and the computing equation changes into:

$$
P R_{i}=s \sum_{j=1}^{N} a_{v} a_{j i} \frac{P R_{j}(k-1)}{k_{j}^{\text {out }}}+\frac{1-s}{N}, i=1,2, \ldots, N
$$

\section{Email Network Model}

The steps of mining important nodes are as follows:

- Compute adjacency matrix $A$ of the directed network.

- $\quad$ Compute metric value $k_{i}$ using (2).

- $\quad$ Normalize and nondimensionalize $k_{i}$ using (3) to get new evaluated matrix.

- Delete interfering nodes using $k$-core algorithm.

- Weight nodes using (7) and get weighted matrix.

- $\quad$ Sort $P R$ values of nodes and mine important nodes. 


\section{EXPERIMENTS}

\section{A. System Design}

An email analysis system based on link mining using Java and myeclipse 10 is developed. System model is shown in Figure1.

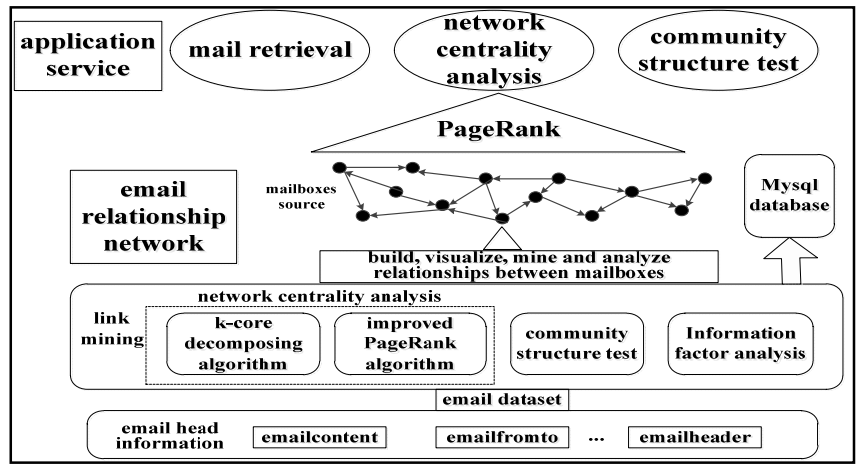

FIGURE I. SYSTEM MODEL

\section{B. Experimental Platform}

Experimental environment is Intel(R) Core(TM) i3-2328M $2.20 \mathrm{GHz}$ processor with $4.00 \mathrm{~GB}(3.25 \mathrm{~GB}$ is usable) RAM, running under the 32 windows7 flagship version of PC.

\section{Experimental Data}

The data in this paper comes from Enron email dataset from computer science website of Carnegie Mellon, including 16052 emails and 151 users.

\section{Experimental Process}

As shown in Figure2, establish and optimize email network then decompose it into layers using $k$-core algorithm, finally mine important nodes using PageRank.

The system provides following methods of extracting relationships in email network:

(1)Starting and ending date. Only emails within the time are considered.

(2)Eliminate isolated nodes. Isolated nodes have no relationships with others.

(3)Core number threshold. Only the nodes whose core numbers are larger than minimum are included, which can delete interferential nodes and optimize the network. In this paper it's 3.

(4)Delete one-way links. Mailboxes are considered only when they connect to each other.

(5)Visualize the nodes in email network finally.

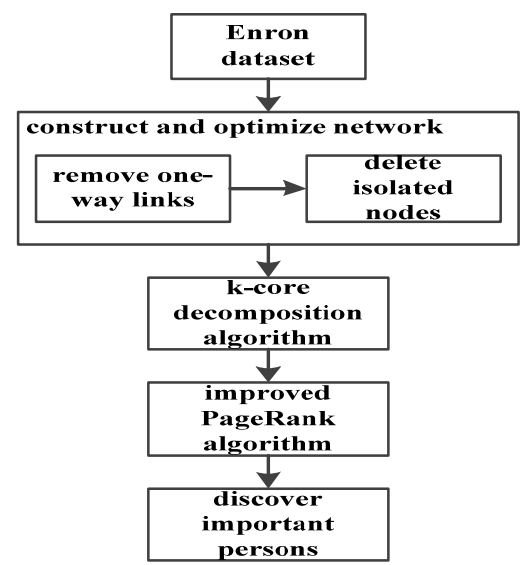

FIGURE II. MINE IMPORTANT PERSONS IN EMAIL NETWORK

\section{E. Experimental Results}

1) Evaluate accuracy using network index: Figure3 shows degree and weight accumulated distribution of nodes. They look like rattails which means the algorithm is power-law distribution function and scale-free. A few nodes with large PR values perform well in robustness.

2) Compare with different methods: Experiments of five mining algorithms are done under the same data. The top five nodes are listed.

\section{a) degree evaluation experiment}

This algorithm counts the number of emails sent and received by nodes. Employees in specific position will have high weights and affect mining important nodes when information flows to them. For instance, Liz Taylor is an interfering node. Results are shown in the Table 1.

b) improved clustering coefficient evaluation experiment

Using clustering coefficient of nodes as metric indexes simply can't describe nodes' importance well. Improve clustering coefficient metric method through combining number of nodes linked to node i and binding factor. But the result isn't steady as shown in Table 2. For example, Philip Allen has high weight mistakenly.

\section{c) EmailRank evaluation experiment}

The importance of nodes depends on other nodes linked directly to them. The result is shown in Table 3.

\section{d) figure entropy theory evaluation experiment}

Weight the degree centrality using entropy. The result showed in Table 4 indicates that it can be affected by interfering nodes. Scott Neal, for example, is an interfering node.

e) improved PageRank algorithm evaluation experiment

Compared with other four experiments, the important members mined in this experiment are more authoritative. Table 5 shows the result. 
TABLE I. DEgRe Evaluation

\begin{tabular}{l|cc} 
ranking & name & position \\
\hline 1 & Sally Beck & COO \\
2 & Liz Taylor & Employee \\
3 & Louise Kitchen & President \\
4 & Kenneth Lay & CEO \\
5 & Michael Grigsby & Manager
\end{tabular}

TABLE II. IMPROVED CLUSTERING COEFFICIENT EVALUATION

\begin{tabular}{c|cc} 
ranking & name & position \\
\hline 1 & Louise Kitchen & President \\
2 & Philip Allen & N/A \\
3 & Barry Tycholiz & Vice President \\
4 & Kevin Presto & Vice President \\
5 & Sally Beck & COO
\end{tabular}

TABLE III. EMAILRANK EVALUATION

\begin{tabular}{c|cc} 
ranking & name & position \\
\hline 1 & Sally Beck & COO \\
2 & Louise Kitchen & President \\
3 & Kenneth Lay & CEO \\
4 & John Lavorato & CEO \\
5 & Michael Grigsby & Manager
\end{tabular}

TABLE IV. FIGURE ENTROPY THEORY EVALUATION

\begin{tabular}{l|cc} 
ranking & name & position \\
\hline 1 & Louise Kitchen & President \\
2 & Mike Grigsby & Manager \\
3 & Greg Whalley & President \\
4 & Scott Neal & Employee \\
5 & Kenneth Lay & CEO
\end{tabular}

TABLE V. IMPROVED PAGERANK ALGORITHM EVALUATION

\begin{tabular}{l|cc} 
ranking & name & position \\
\hline 1 & Louise Kitchen & President \\
2 & Barry Tycholiz & Vice President \\
3 & Kevin Presto & Vice President \\
4 & Sally Beck & COO \\
5 & Greg Whalley & President
\end{tabular}

It's found that Louise Kitchen and Greg Whalley are former presidents of Enron Company. They are the most important nodes. Barry Tycholi and Kevin Presto are former vicepresidents of Enron Company so they take second place. Sally Beck is former $\mathrm{COO}$ (Chief Operating Officer) and information circulates quickly at this node.

\section{CONCLUSIONS}

In order to solve the problem that nodes with large degrees may interfere others in evaluating important nodes in the email network. This paper proposes a method based on social network centrality evaluation. By deleting interfering nodes from outer layers, it can reduce the computational complexity of PageRank algorithm.

This paper also improves PageRank algorithm by distributing $P R$ values unevenly.

Through analyzing experimental results, comparing with other evaluation algorithms and visualizing, it's validated that the improved PageRank algorithm and email network analysis system based on link mining proposed in this paper do well in mining important members in the email network.

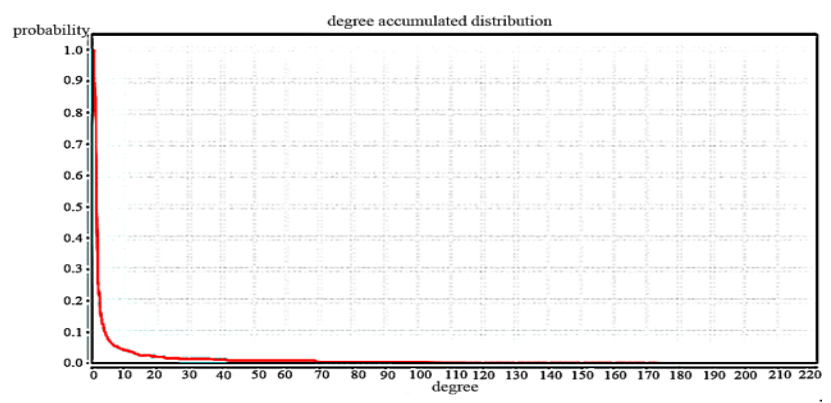

(a)degree accumulated distribution

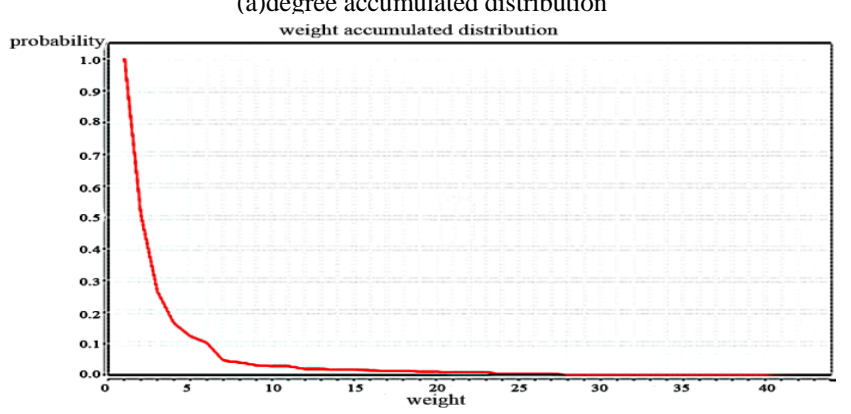

(b)weight accumulated distribution

FIGURE III. STATISTICAL DISTRIBUTION

\section{ACKNOWLEDGMENT}

The work has been supported by project (U1536116) funded by National Natural Science Foundation of China (NSFC).

\section{REFERENCES}

[1] Bisharat Rasool Memon,“ Identifying Important Nodes in Weighted Covert Networks using Generalized Centrality Measures,” EISIC. 2012, pp. 131-140.

[2] F Hu, Y Liu, J Jin,“ Multi-index Evaluation Algorithm Based on Locally Linear Embedding for the Node Importance in Complex Networks," DCABES. 2014, pp. 138-142.

[3] Xiangju Li, Hong Zhao, William Zhu, “ A cost sensitive decision tree algorithm with two adaptive mechanisms,”Knowl.-Based Syst. Vol. 88 (2015), pp. 24-33.

[4] S.Huang, H.F.Cui, Y.M.Ding, “ Evaluation of node importance in complex networks,” arXiv: 1402.5743.

[5] Lu Zhong, Chao Gao, Zili Zhang, Ning Shi, “ Identifying Influential Nodes in Complex Networks for Network Immunization,” JCIS. Vol. 10 No.20 (2014), pp. 8767-8774.

[6] Teng Wang, Yanni Han, Jie Wu, “ Evaluate Nodes Importance In Directed Network Using Topological Potential,” ICIECS. 2010, pp. 1- 4.

[7] Liang Sun, Hongwei Ge, Xiaoli Guo, “ An Algorithm with User Ranking for Measuring and Discovering Important Nodes in Social Networks,” BMEI. 2014, pp. 945 - 949.

[8] Kazumi Saito, Masahiro Kimura, Kouzou Ohara, Hiroshi Motoda, “ Super mediator-A new centrality measure of node importance for information diffusion over social network,” Inform.Sci. Vol. 329 (2016), pp. 9851000.

[9] Jianshe Wu, Fang Wang, Peng Xiang, “ utomatic network clustering via density-constrained optimization with grouping operator,” Applied Soft Computing Vol. 38 (2016), pp. 606-616.

[10] Jitesh Shetty, Jafar Adibi, “ Discovering Important Nodes through Graph Entropy The Case of Enron Email Database,” Proc. of LinkKDD. 2005. 
[11] Peter Lofgren, Ashish Goel, “ Personalized PageRank to a Target Node,” arXiv: 1304.4658.

[12] Jessica Liebig, Asha Rao: “ Identifying Influential Nodes in Bipartite Networks Using the Clustering Coefficient,” SITIS. 2015, pp. 323-330.

[13] Shinjae Yoo, Yiming Yang, Frank Lin, II-Chul Moon, “ Mining Social Networks for Personalized Email Prioritization,” KDD. 2009, pp. 967976.

[14] Sergio Crisostomo, Udo Schilcher, Christian Bettstetter, Joao Barros, "Probabilistic flooding in stochastic networks:Analysis of global information outreach,” Computer Networks Vol. 56 (2012), pp. 142-156.

[15] Sancheng Peng, Min Wu, Guojun Wang, Shui Yu, "Containing smartphone worm propagation with an influence maximization algorithm,” Computer Networks Vol. 74 (2014), pp. 103-113.

[16] Pei Wang, Xinghuo Yu, Jinhu Lü, Aimin Chen, “ Identification of Important Nodes In Artificial Bio-Molecular Networks”, ISCAS. 2014, pp. 1267-127. 